Cabero Almenara, J.; Marín-Díaz, V. y Sampedro-Requena, B.E. (2018). Aceptación del Modelo Tecnológico en la enseñanza superior. Revista de Investigación Educativa, 36(2), 435-453.

DOI: http://dx.doi.org/10.6018/rie.36.2.292951

\title{
Aceptación del Modelo Tecnológico en la enseñanza superior'
}

\author{
Technology Acceptance Model \\ in Higher Education
}

\author{
Julio Cabero Almenara*,Verónica Marín-Díaz y Begoña E. Sampedro-Requena*** \\ "Dpto. Didáctica y Organización Educativa. Facultad de Ciencias de la Educación. Universidad de Sevilla (España) \\ * Dpto. Educación. Facultad de Ciencias de la Educación. Universidad de Córdoba (España) \\ * Dpto. Educación. Facultad de Ciencias de la Educación. Universidad de Córdoba (España
}

\begin{abstract}
Resumen
En las últimas décadas la incorporación de las plataformas de teleformación a la educación superior ha supuesto que los alumnos convivan con un elemento ajeno a su vida social, por lo que su implicación con ella depende de un gran número de factores. A través del Modelo de Aceptación Tecnológica (TAM) de Arteaga y Duarte (2010), ideado originalmente por Davis en 1989, el cual se basa en la teoría psicológica de Azjen y Fishbein (1980), se ha procedido a recabar las opiniones que los estudiantes de Grado de Educación Primaria de la Universidad de Córdoba $(N=546)$ tienen sobre Moodle, -plataforma para desarrollar la formación online en dicha universidad-, por medio del establecimiento de 23 hipótesis. El principal resultado ha sido constatar, que el modelo anglosajón puede ser aplicado en el contexto español sin riesgo de pérdida o sesgo de información. Por otra parte, se ha encontrado que el sexo no es una variable predictora del éxito/fracaso en la aceptación de esta tecnología, al igual que sucede con la for-
\end{abstract}

1 Artículo resultado del proyecto de investigación I+D concedido por el Ministerio de Ciencia e Innovación del Gobierno de España (clave: EDU2014_57446P) denominado "Realidad Aumentada para Aumentar la Formación, (RAFODIUN)".

Correspondencia: Verónica Marin-Díaz, vmarin@uco.es; Dpto. Educación. Facultad de Ciencias de la Educación. Universidad de Córdoba (España). 
mación previa que tengan los estudiantes. El empleo del modelo TAM a la educación superior española revertirá en el uso de una forma más eficaz de las tecnologías emergentes que día a día se van incorporando a la docencia universitaria.

Palabras clave: enseñanza superior, motivación, estudiante universitario, moodle.

\section{Abstract}

In the last decades, the incorporation of online learning platforms to higher education means that students live with an unknown element in their social lives. So, their involvement with technologies depends on a number of factors. Through the Technology Acceptance Model (TAM) of Arteaga and Duarte (2010), created by Devis (1984), which is based on the psychological theory of Azjen and Fishbein (1980), the opinions of primary education degree seeking students at the University of Cordoba $(N=546)$ were collected. Specifically, their opinions on Moodle, a platform to develop online training at said university, through the establishment of 23 working hypotheses. The main results have been confirmed that the English model can be applied in a Spanish context without risk of loss or information bias. On the other hand, it has been found that sex is not a predictor of success or failure in the acceptance of this technology nor the previous training that a student has. The use of TAM in Spanish higher education will revert to a more efficient way of emerging technologies that are incorporated more and more everyday into university teaching.

Keywords: higher education, motivation, student, moodle.

\section{Introducción}

La reforma universitaria de los títulos académicos ha traído consigo la reformulación de las metodologías de aula, más allá de la simple modificación de los contenidos curriculares de las materias propuestas (Herrero, González \& Marín, 2015). Estos cambios en los procesos de enseñanza, han afectado de manera directa a la forma en que los estudiantes universitarios desarrollan su proceso de aprendizaje. Además, han puesto sobre la mesa la incorporación de manera latente de las herramientas que hoy hay disponibles en la red de redes, Internet, en concreto nos referimos a las plataformas de teleformación o LMS, y dentro de ellas a Moodle.

Hasta ahora han sido variadas las investigaciones realizadas en torno al grado de uso y aceptación tecnológica de los docentes y discentes universitarios (Arteaga \& Duarte, 2010; Colorado, Marín \& Zavala, 2016; Marín, Ramírez \& Maldonado, 2016) así como las recomendaciones sobre la necesidad de incorporar las tecnologías a la enseñanza universitaria, sin embargo no se han centrado en una herramienta concreta, siendo estudios de índole generalista. Como señalan Padilla-Meléndez, del Águila-Obra y Garrido-Moreno (2015) y Gámiz y Gallego (2016) la formación de los estudiantes del nuevo sistema de enseñanza superior no supone per sé, que los elementos de índole digital como son las LMS supongan una mejora en el proceso de aprendizaje de los estudiantes; no obstante, son elementos sobre los que el diseño de los títulos universitarios actuales giran. Es por ello, que consideramos necesario el estudio de la adopción tecnológica respecto a Moodle que presenta el alumnado universitario; en este trabajo presentamos los resultados que muestran los estudiantes de Grado de Educación Primaria de la Universidad de Córdoba en torno a su adopción de las tecnologías, en concreto a Moodle. 


\section{EI Modelo TAM de análisis de adopción de tecnologías}

En 1989 Davis formuló el modelo Technology Acceptance Model o más conocido como TAM, a partir del trabajo de Ajzen y Fishbein (1980) sobre la teoría psicológica de "Acción Razonada", la cual sugiere que las intenciones y actitudes de una persona, pueden ser de utilidad para predecir la conducta que mostrará una persona, y la de Bandura (1990) "autoeficacia percibida". El modelo TAM nos sugiere que la aceptación de cualquier tecnología por una persona, vendrá influida por las creencias que tenga sobre las consecuencias de su utilización, tanto desde un punto de vista personal, como laboral, profesional o académico.

En la formulación inicial del modelo TAM por Davis, se indica que la actitud que se tenga hacia el uso de cualquier "Tecnología de la Información y Comunicación" vendrá determinada por dos variables específicas, de un lado la utilidad percibida (Perceived Usefulness) y de otro la facilidad de uso percibida (Perceived Ease of Use) (ver figura 1); teniendo ambas una consecuencia directa sobre la actitud que la persona tenga hacia el uso o no de la tecnología analizada. Entendiendo Davis (1989, p.320) por utilidad percibida una motivación extrínseca de la persona y definiéndola como "la probabilidad subjetiva de una persona de que, al usar un determinado sistema, mejorará su actuación en el trabajo"; y por facilidad de uso percibido: "grado por el que una persona cree que usar un determinado sistema estará libre de esfuerzo", el modelo TAM, como señalan Peral-Peral, Arenas y Ramón-Jerónimo (2014), sugiere que la aceptación de la tecnología por parte de un individuo está influida por las creencias que este tiene sobre las consecuencias de su uso. TAM predice que los usuarios adoptan una nueva tecnología cuando sus percepciones de la facilidad de uso y la utilidad de la tecnología son positivas (Peral-Peral et al., 2014, p. 44).

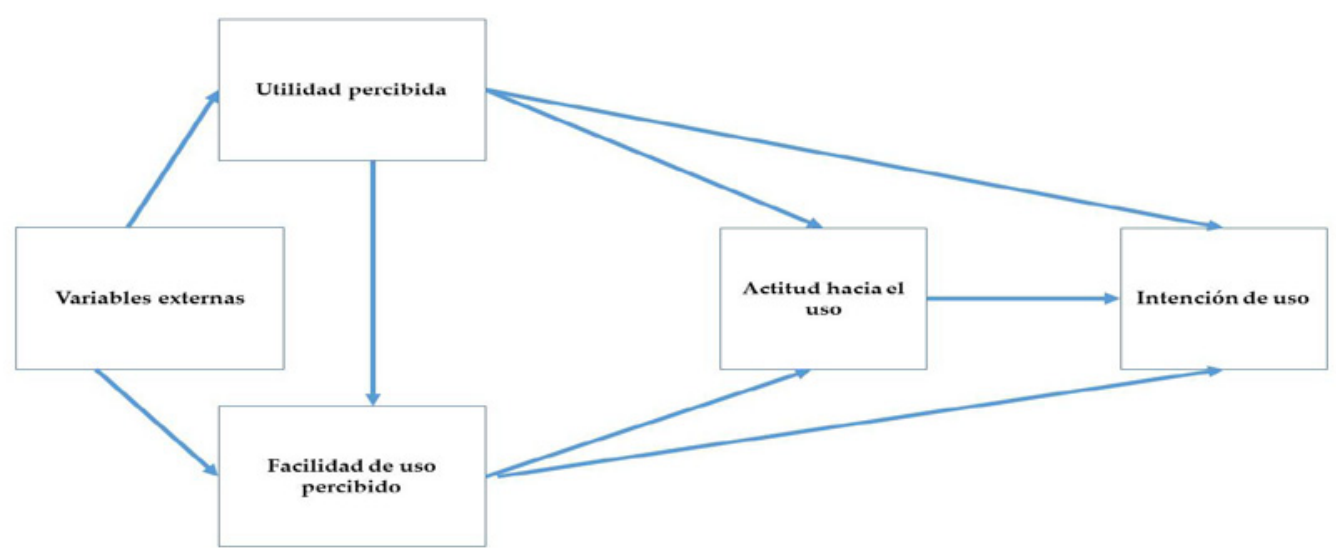

Figura 1. Formulación inicial de TAM (Davis, 1989).

Señalemos desde el principio dos aspectos: a) que a pesar de que TAM ha ido evolucionando a lo largo del tiempo (Escobar, Carvajal \& Monge, 2014; López-Bonilla \& López-Bonilla, 2011; Park, Saplan \& Jaegal, 2012), el modelo sigue estando constituido 
en su núcleo por las variables por el conjunto simple de variables identificadas por su creador y ello probablemente su mayor ventaja, y b) como han puesto de manifiesto algunos meta-análisis realizados sobre las investigaciones que lo han utilizado (LópezBonilla \& López-Bonilla, 2011) se muestra como un modelo confiable para analizar la actitud y la intención de uso hacia una tecnología. De todas formas, y como llaman la atención López-Bonilla y López-Bonilla (2011), la aplicación del modelo TAM requiere de su conceptualización específica para la investigación concreta que se lleve a cabo, lo que implica que los instrumentos de análisis utilizados deben ser contextualizados y por tanto, generados para cada estudio.

El modelo también nos sugiere que determinadas variables externas pueden servir de predictoras, $y$, por tanto, incidir en la utilidad y la facilidad de uso percibidas por las personas respecto a la tecnología (Yong, Rivas \& Chaparro, 2010). Distintos trabajos han analizado específicamente algunas de estas variables entre las que se encuentran: nivel de formación, tipo de usuario, experiencia en el manejo de tecnologías, género, soporte técnico de ayuda, edad, o nivel profesional (Arenas, Peral-Peral \& RamónJerónimo, 2014; Arteaga \& Duarte, 2010; Bao, Xong, Hu \& Kibelloh, 2013; Hsiao \& Yang, 2011; Kumar \& Kumar, 2013; López-Bonilla \& López-Bonilla, 2011; Terzis \& Economides, 2011).

El modelo TAM ha sido utilizado, tanto para conocer el grado de adopción de las tecnologías por parte de diferentes colectivos, como de tecnologías específicas utilizadas. En el primero de los casos, y poniendo el punto de atención en la educación, encontramos trabajos centrados en los docentes y discentes (Teo, Fan \& Du, 2015; Valencia, Benjumea \& Rodríguez, 2014; Yong et al., 2010), y en los directivos y administradores del centro (González-Bravo \& Valdivia-Peralta, 2015). Con respecto al segundo, se han llevado a cabo trabajos, tanto para analizar las percepciones de uso general hacia las tecnologías (González-Bravo \& Valdivia-Peralta, 2015), como a tecnologías específicas. En este último caso se han efectuado investigaciones para analizar el grado de adopción de tecnologías como son: los portafolios (Wai-tsz, Chi-kin, Chang, Zhang \& Chiu, 2014), los ordenadores (Aguilar-Luzón, Berrios-Martos \& López-Zafra, 2012; Silva, Pimentel \& Soares, 2012), las bibliotecas virtuales (Chen \& Chengalur-Smith, 2015), los e-book (Jin, 2014), la computación en nube (Jou \& Wang, 2013), las comunidades virtuales (Liu, Chang, Sun, Wible \& Kuo, 2010), video plataformas como Youtube (Lee \& Lehto, 2013), los dispositivos móviles (Chen, Sivo, Seilhamer, Sugar \& Mao, 2013; Park, Nam \& Cha, 2012;), los videojuegos aplicados a la educación (Cheng, Lou, Kuo \& Shih, 2013), la realidad aumentada (Cabero \& Barroso, 2016), o las redes sociales (Marín, Sampedro \& Muñoz, 2015).

Lógicamente estos estudios han llegado también al análisis de la modalidad educativa del e-learning (Kang \& Shin, 2015; Mohammadi, 2015; Park, Nam et al., 2012; Persico, Manca \& Pozzi, 2014; Tarhini, Hone \& Liu, 2014; Valencia et al., 2014). Y dentro de ella han efectuado trabajos para analizar las actitudes hacia el uso de los LMS o plataformas de formación virtual de manera general, como el trabajo de Alharbi y Drew (2014), o específica, como los realizados por Ngai, Poon y Chan (2007) y Sánchez-Franco (2010) respecto a la WebCT, o el de Arteaga y Duarte (2010) sobre Moodle.

Para finalizar este apartado podemos señalar que el modelo TAM presenta un alto interés en la literatura científica y en la investigación sobre el terreno de las TIC (Hsiao \& Yang, 2011; Schoonenboom, 2014). 


\section{Método}

\section{El modelo TAM utilizado e hipótesis}

El modelo TAM desarrollado para la investigación se presenta en la figura 2, donde se exponen las grandes variables del modelo y las variables externas que se han considerado en el trabajo. Modelo que de forma más elemental fue utilizado por Arteaga y Duarte (2010) en su trabajo.

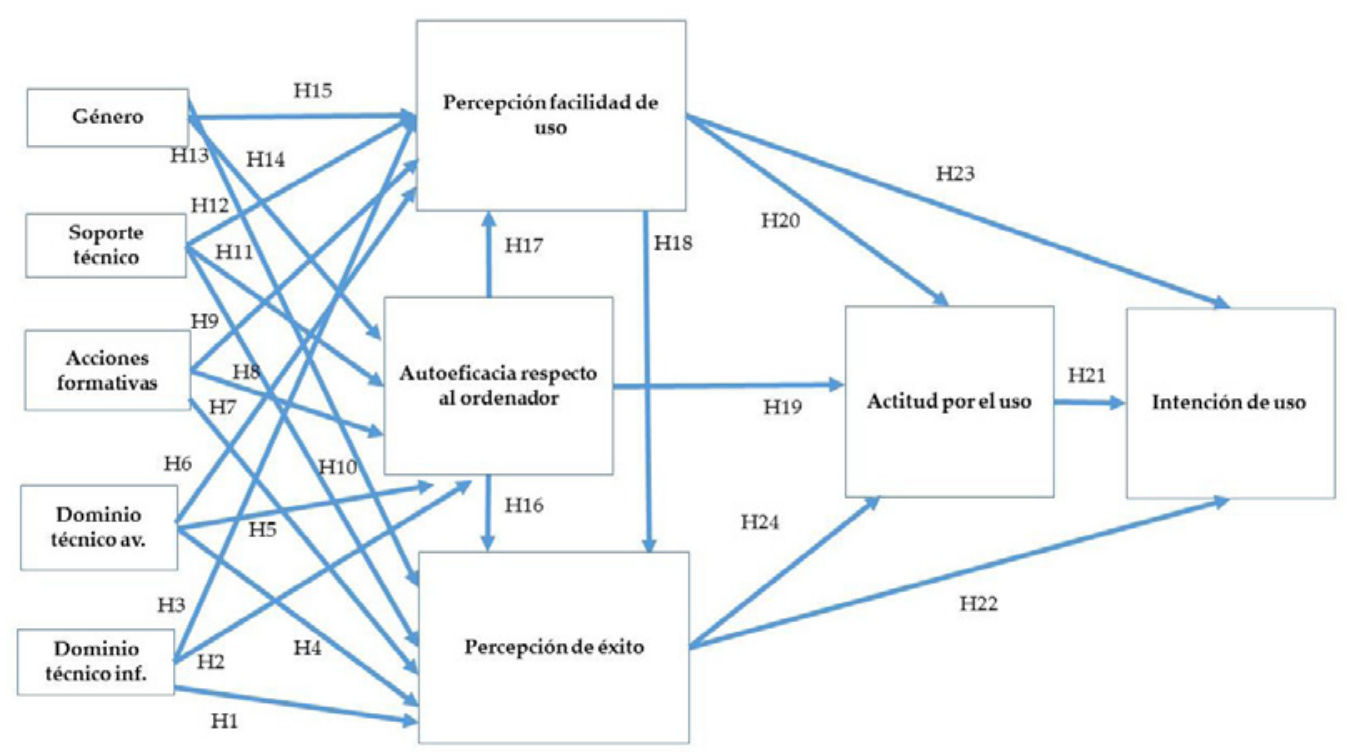

Figura 2. Modelo TAM.

Del modelo se desprenden 23 hipótesis, que intentaremos contrastar en nuestro trabajo y que las declaramos en los siguientes términos:

H1-H2-H3: El dominio técnico que el alumno indica tener de los ordenadores tiene un efecto positivo sobre la percepción de éxito hacia el uso del Moodle, sobre la autoeficacia percibida respecto al uso del Moodle, y sobre la percepción de facilidad de uso hacia el uso del Moodle.

H4-H4-H5: El dominio técnico que el alumno indica tener de los medios audiovisuales tiene un efecto positivo sobre la percepción de éxito hacia el uso del Moodle, sobre la autoeficacia percibida respecto al uso del Moodle y sobre la percepción de facilidad de uso hacia el uso del Moodle.

H7-H8-H9: El haber realizado acciones formativas para el aprendizaje de alguna tecnología tiene un efecto positivo sobre la percepción de éxito hacia el uso del Moodle, sobre la autoeficacia percibida respecto al uso del Moodle, y sobre la percepción de facilidad de uso hacia el uso del Moodle. 
H10-H11-H12: El disponer de un soporte técnico de apoyo tiene un efecto positivo sobre la percepción de éxito hacia el uso del Moodle, sobre la autoeficacia percibida respecto al uso del Moodle, y sobre la percepción de facilidad de uso hacia el uso del Moodle.

H13-H14-H-15: El género del alumno tiene un efecto positivo sobre la percepción de éxito hacia el uso del Moodle, sobre la autoeficacia percibida respecto al uso del Moodle, y sobre la percepción de facilidad de uso hacia el uso del Moodle.

H16: La autoeficacia percibida del alumno respecto al ordenador tiene un efecto positivo sobre la percepción de éxito en el uso del Moodle.

H17: La autoeficacia percibida del alumno respecto al ordenador tiene un efecto positivo sobre la percepción de facilidad de uso hacia el uso del Moodle.

H18: La percepción de facilidad de uso del alumno tiene un efecto positivo sobre la percepción de éxito hacia el uso del Moodle.

H19: La autoeficacia percibida del alumno respecto al ordenador tiene un efecto positivo sobre la actitud hacia el uso del Moodle.

H20: La percepción de facilidad de uso del alumno tiene un efecto positivo sobre la actitud hacia el uso del Moodle.

H21: La actitud hacia el uso del Moodle tiene un efecto positivo sobre la intención de uso del Moodle.

H22: La percepción de éxito del alumno tiene un efecto positivo sobre la intención de uso del Moodle, y sobre la actitud hacia el uso del Moodle.

H23: La percepción de facilidad de uso del alumno tiene un efecto positivo sobre la intención de uso del Moodle.

Para la justificación de la selección de las variables externas nos hemos apoyado en las investigaciones que se señalan en la tabla 1, donde las mismas se mostraron como significativas.

Tabla 1

Variables externas consideradas en nuestro estudio

\begin{tabular}{ll}
\hline \multicolumn{1}{c}{ Variables externas } & \multicolumn{1}{c}{ Autores } \\
\hline Género & $\begin{array}{l}\text { Ho, Hung y Chen, 2013; Hohlfeld, Ritzhaupt y Barron, 2013; } \\
\text { Olaoluwakotansibe, 2013; Tarhini et al., 2014; Yong et al., } \\
\text { 2010. }\end{array}$ \\
Soporte técnico & APS Group Scotland, 2015; Arteaga y Duarte, 2010. \\
Acciones formativas & $\begin{array}{l}\text { Padilla-Meléndez et al., 2015; Tarhini et al., 2014; Yong et al., } \\
\text { 2010. }\end{array}$ \\
Dominio técnico audiovisual & Cabero, Leal, Andrés y Llorente, 2009. \\
Dominio técnico informática & Cabero et al., 2009; Tarhini et al., 2014. \\
\hline
\end{tabular}




\section{Instrumento de recogida de información}

El instrumento utilizado para el análisis del TAM se basa en el elaborado por Arteaga y Duarte (2010), el cual estaba conformado por 28 ítems que recogen información de seis grandes dimensiones: soporte técnico (ST) (6 ítems), percepción de éxito (PE) (6 ítems), autoeficacia respecto al ordenador (AO) (6 ítems), percepción facilidad de uso (FUP) (4 ítems), actitud hacia el uso (A) (4 ítems), y uso del sistema (US) (2 ítems). Señalar que está construido con formato de respuesta tipo Likert, con siete opciones de respuesta (1=Extremadamente improbable/en desacuerdo a 7=Extremadamente probable/de acuerdo) (Anexo), y se administró vía Internet.

$\mathrm{Su}$ fiabilidad se obtuvo mediante la alfa de Cronbach, que según O'Dwyer y Bernauer (2014) es lo más apropiado para este tipo de instrumentos, obteniéndose los siguientes valores: total del instrumento .901; por factores ST (.811), PE (.874), $\mathrm{AO}(.652)$, FUP (.809), AU (.777) y US (.677). Valores que de acuerdo con diferentes autores (George \& Mallery, 2009; Mateo, 2004), nos permiten considerarlas como "moderadas" en las dimensiones "AO" y "US" y "altas" en el resto. También llevamos a cabo la correlación ítem-total, para analizar si eliminando algún ítem aumentaría la fiabilidad del instrumento, que no exponemos para no extendernos, pero si queremos señalar que los resultados obtenidos no reflejaron la necesidad de prescindir de ningún ítem.

Por otro lado, el análisis factorial exploratorio realizado de los componentes principales, arrojó que había que extraer cinco factores: soporte técnico (TS), percepción de éxito (PE), autoeficacia respecto al ordenador $(\mathrm{AO})$, percepción facilidad de uso (FUP) y uso del sistema (US), dado que la variable actitud hacia el uso (A) carga en percepción de éxito (PE), por lo que eliminamos esta variable a partir de nuestro modelo. Asimismo, se prescindió de los siguientes elementos, respecto a la dimensión soporte técnico (ST): ST1 y ST6; y de la autoeficacia respecto al ordenador (AO): $\mathrm{AO} 1, \mathrm{AO} 2, \mathrm{AO} 5$ y AO6, debido a que cargaban en distintos componentes, lo cual no coincide con otros estudios (Arteaga \& Duarte, 2010), que explican el soporte técnico y la autoeficacia con el ordenador; quizás sea debido a que los sujetos participantes no tenían conocimiento de la posible asistencia del sistema Moodle o a su poca destreza personal con el mismo.

En un segundo momento, al realizar el análisis factorial confirmatorio, después de aplicados los cambios, el índice KMO (Kaiser-Meyer-Olkin) fue de .898, lo cual sugiere que los datos están suficientemente interrelacionados y que el análisis factorial es factible. Los cinco componentes obtenidos explican el $61.13 \%$ de la varianza total, cuyos resultados aparecen en la tabla 2. Valores que al mismo tiempo eran similares a los alcanzados en su investigación por Arteaga y Duarte (2010), los cuales se situaron en el .834 y el $76.23 \%$ respectivamente.

La muestra estuvo formada 546 sujetos, de los cuales el $31.1 \%$ son hombres y el $68.9 \%$ mujeres, de los cuales el $17.12 \%$ cursaban el Grado de Educación Infantil y el 82,2\% el de Educación Primaria (82.8\%), en el Centro de Magisterio “Sagrado Corazón" (13.9\%) adscrito a la Universidad de Córdoba y en la Facultad de Ciencias de la Educación de citada universidad (86.1\%). Señalar que el 37\% se encuentran cursando primero y el 63\% segundo. 
Tabla 2

Resultado del análisis de los componentes principales

\begin{tabular}{|c|c|c|c|c|c|}
\hline & \multicolumn{5}{|c|}{ Componentes } \\
\hline & $\begin{array}{c}\text { Factor } 1 \\
\text { percepción } \\
\text { de éxito }\end{array}$ & $\begin{array}{c}\text { Factor } 2 \\
\text { percepción } \\
\text { facilidad de } \\
\text { uso }\end{array}$ & $\begin{array}{l}\text { Factor } 3 \\
\text { soporte } \\
\text { técnico }\end{array}$ & $\begin{array}{c}\text { Factor } 4 \\
\text { uso del } \\
\text { sistema }\end{array}$ & $\begin{array}{c}\text { Factor } 5 \\
\text { autoeficacia } \\
\text { respecto al } \\
\text { ordenador }\end{array}$ \\
\hline PE_1 & .670 & & & & \\
\hline PE_2 & .775 & & & & \\
\hline PE_3 & .787 & & & & \\
\hline PE_4 & .772 & & & & \\
\hline PE_5 & .674 & & & & \\
\hline PE_6 & .748 & & & & \\
\hline FUP_1 & & .739 & & & \\
\hline FUP_2 & & .790 & & & \\
\hline FUP_3 & & .649 & & & \\
\hline FUP_4 & & .766 & & & \\
\hline ST_2 & & & .725 & & \\
\hline ST_3 & & & .722 & & \\
\hline ST_4 & & & .646 & & \\
\hline ST_5 & & & .628 & & \\
\hline US_1 & & & & .697 & \\
\hline US_2 & & & & .803 & \\
\hline AO_3 & & & & & .556 \\
\hline $\mathrm{AO} \_4$ & & & & & .761 \\
\hline Alfa de Cronbach & .872 & .814 & .811 & .700 & .689 \\
\hline Eigenvalue & 3.67 & 2.58 & 1.99 & 1.44 & 1.31 \\
\hline $\begin{array}{l}\text { Varianza explicada } \\
\text { acumulada \% }\end{array}$ & 61.25 & 64.49 & 49.89 & 72.06 & 65.52 \\
\hline
\end{tabular}

\section{Descripción de la muestra}

Respecto a las actividades formativas realizadas por estos estudiantes para el aprendizaje de las tecnologías de la información y la comunicación (TIC), el 71.2\% expresan no haberlas efectuado frente al $28.8 \%$ que manifiestan afirmativamente este hecho.

El 14.5\% indican una valoración suficiente en la formación personal para el manejo técnico de medios audiovisuales frente al $0.7 \%$ que señalan nada y casi nada (ambos). Mientras que el 1.6\% indican muchísimo; el 5.7\% casi mucho; el 15.4\% bastante; el 26.2\% casi bastante; el 22.5\% muy suficiente. Por el contrario, el 2.2\% realizan una valoración 
personal de muy poco en esta manipulación; el 4.6\% poco; y el 5.9\% algo. Asimismo, la valoración que indican en su formación personal para el manejo técnico de los ordenadores, atiende a la siguiente distribución: el $0.4 \%$ nada; el $0.4 \%$ casi nada; el 1.5\% muy poco; el 5.5\% poco; el 5.5\% algo; el 13.9\% suficiente; el 22.3\% muy suficiente; $25.6 \%$ casi bastante; el 16.5\% bastante; el 6.0\% casi mucho; y 2.4\% muchísimo. Finalmente, el 20.5\% de los estudiantes manifiestan una valoración muy suficiente en su formación personal para el manejo técnico de Internet; el 29.5\% casi bastante; el 20.7\% bastante; el $9.9 \%$ casi mucho; y el $2.4 \%$ muchísimo; frente al $0.2 \%$ que indican nada; el $0.5 \%$ casi nada; el 1.1\% muy poco; el 1.8\% poco; y $4.4 \%$ algo. Solo el $9 \%$ consideran suficiente su formación personal en la manipulación técnica de Internet.

\section{Resultados}

Las medias y desviaciones típicas alcanzadas tanto para la globalidad del instrumento como sus diferentes dimensiones fueron: total del instrumento: $\mathrm{M}=5.18 \mathrm{DT}=.65$; ST: $M=4.75$ DT=.91 CURTOSIS=.66; PE: $M=5.49$ DT=.88 CURTOSIS=1.93; AO: $M=5.06$ DT=.74 CURTOSIS=1.23; FUP: $M=5.40$ DT=.93 CURTOSIS=1.60; AU: $M=5.41$ DT=.94 CURTOSIS=1.34; US: M=4.92 DT=1.10 CURTOSIS=-.13, como indica García-Lirios (2012) se puede establecer la distribución normal mediante el estadístico de la curtosis cuyo valores deben oscilar entre $-3 \mathrm{y}+3$, por lo que los valores obtenidos se encuentran en este umbral requerido de distribución normal.

Los valores medios obtenidos tienden a situarse un punto y medio por encima del valor central de 3.5, lo que indica un cierto grado de acuerdo de los alumnos en la diversidad de ítems que le fueron presentados. Indicar que las desviaciones típicas logrados muestran cierta variabilidad en las contestaciones ofrecidas por los estudiantes. En la tabla 3, se presentan las medias y desviaciones típicas alcanzadas en todos los ítems.

Las medias alcanzadas permiten señalar: que el soporte técnico en el que se apoya Moodle ni dificulta ni facilita el uso del mismo, que es ligeramente probable que pueda ser útil para aumentar su conocimiento sobre esta plataforma, asimismo, ligeramente probable que pueda ser ventajoso para acrecentar el propio manejo con el ordenador, que es ligeramente factible la percepción de facilidad en el uso de la misma, que es ligeramente posible desarrollar actitudes positivas hacia su empleo y, permite un uso regular del sistema. En definitiva, que pueden hacer "más fácil aprender en la universidad" (5.54) y "es una buena idea" (5.84).

Respecto al género, la hipótesis nula (H0) formulada se refiere a si éste no tiene una influencia significativa con un riesgo alfa de equivocarnos del .05, en la percepción de éxito, la percepción de facilidad de uso y en la autoeficacia respecto al ordenador. Para su contraste aplicamos el estadístico t de Student, el cual puede utilizarse si se exceden los 120 grados de libertad (Hernández, Fernández y Baptista, 2014), que para 544 gl dieron los siguientes valores y nivel de significación: PE: $\mathrm{t}=-1.445$ ( $p=.149)$; FUP: $\mathrm{t}=-.862(p=.389) ; \mathrm{y}$ AO: $\mathrm{t}=-1.207(p=.288)$.

Valores que no permiten rechazar ninguna de las $\mathrm{H} 0$ formuladas con un riesgo alfa de equivocarnos igual o inferior al .05; en consecuencia podemos decir que el género de los estudiantes no influye en la percepción de éxito, la percepción de facilidad de uso de Moodle y en la autoeficacia respecto al ordenador. Asimismo, al considerar las 
Tabla 3

Medias y desviaciones típicas obtenidos en los diferentes items del instrumento

\begin{tabular}{l}
\hline Ítem \\
\hline 1. El sistema provee de asistencia cuando hay un problema técnico (ST1) \\
2. Hay una línea telefónica. disponible en cualquier momento (ST2) \\
3. Se puede preguntar por fax cuando hay algún problema técnico (ST3) \\
4. Se puede preguntar por correo electrónico cuando hay un problema técnico \\
(ST4)
\end{tabular}

5. Se pueden hacer preguntas a través de la web cuando hay un problema técnico (ST5)

6. El sistema ofrece un buen soporte técnico (ST6)

M. D.T.

$4.77 \quad 1.325$

4.511 .629

3.741 .476

5.291 .418

7. El sistema basado en la Web me ayuda a aprender más eficazmente (PE1)

4.991 .456

8. El sistema web mejora mi desempeño académico (PE2)

$5.20 \quad 1.161$

5.401 .168

9. El sistema basado en la Web hace mi aprendizaje más efectivo (PE3)

$5.49 \quad 1.151$

10. El sistema basado en la Web hace más fácil aprender en la universidad (PE4)

5.411 .109

11. El sistema basado en la Web me da más control en todo mi aprendizaje (PE5)

$5.54 \quad 1.159$

12. En general. el sistema basado en la Web es una ventaja para mi aprendizaje (PE6)

$5.36 \quad 1.134$

5.711 .008

13. Puedo acceder al contenido alojado en el sistema basado en la Web (AO1)

$5.79 \quad 1.041$

14. Puedo navegar libremente por los contenidos alojados en el sistema basado en la Web (AO2)

$5.45 \quad 1.238$

15. Puedo usar el sistema basado en la Web sin necesidad de saber cómo funciona (AO3)

$4.33 \quad 1.401$

16. Puedo resolver los problemas que pueden surgir en el sistema basado en la Web (AO4)

4.111 .462

17. Puedo utilizar el sistema basado en la Web si hay manuales disponibles (AO5)

$4.97 \quad 1.187$

18. En general. estoy en condiciones de utilizar el sistema basado en la Web (AO6)

19. Aprender a usar el sistema basado en la Web es fácil para mí (PFU1)

$5.70 \quad .988$

20. Es fácil conseguir materiales del sistema basado en la Web (PFU2)

$5.46 \quad 1.225$

21. El proceso de uso del sistema basado en la Web es claro y comprensible (PFU3)

$5.40 \quad 1.174$

22. En general. creo que el sistema basado en Web es fácil de usar/utilizar (PFU4)

5.291 .153

23. El aprendizaje basado en la Web es divertido (A1)

$5.44 \quad 1.104$

24. Utilizar el sistema basado en la Web es una buena idea (A2)

$4.71 \quad 1.395$

25. El sistema basado en la Web es un camino atractivo hacia el aprendizaje (A3)

$5.84 \quad 1.090$

26. En general. me gusta utilizar/usar el sistema basado en la Web (A4)

5.561 .109

27. Utilizo el sistema web. en una escala donde 1 es nunca y 7 es mucho (US1)

$5.51 \quad 1.207$

28. El número de horas que empleo en el sistema web es en una escala de 1 es nunca y 7 es muchas horas (US2) 
acciones formativas, la hipótesis nula (H0) expresada referida a si éste no tiene una influencia significativa con un riesgo alfa de equivocarnos del 0.05, en la percepción de éxito, la percepción de facilidad de uso y en la autoeficacia respecto al ordenador, revela en el contraste mediante la aplicación del estadístico $t$ de Student, que para 544 gl: PE: $\mathbf{t}=-.930$ ( $p=.353) ;$ FUP: $\mathrm{t}=-.336$ ( $p=.737) ; \mathrm{y}$ AO: $\mathrm{t}=-.297$ ( $p=.767)$. Lo que no nos permitía rechazar las $\mathrm{H} 0$ contrastadas.

Estos resultados no permiten rechazar ninguna de las hipótesis nulas formuladas, con un riesgo alfa de equivocarnos igual o inferior al .05; en consecuencia podemos decir que las acciones formativas realizadas por el alumnado no influyen en la percepción de éxito, la percepción de facilidad de uso de Moodle y en la autoeficacia respecto al ordenador.

Por otro lado, el dominio técnico que el alumnado indica tener de los ordenadores, formulada la hipótesis nula (H0) equivalente a no poseer una influencia significativa, con un riesgo alfa de equivocarnos del 0.05 y aplicando el estadístico de ANOVA, en la percepción de éxito $\mathrm{F}(10.535)=4.497$ y $p=.000, \mathrm{~h}^{2}=.08$; la percepción de facilidad de uso $\mathrm{F}(10.535)=6.170$ y $p=.000, \mathrm{~h}^{2}=.10$; y en la autoeficacia respecto al ordenador $\mathrm{F}(10.535)=5.560$ y $p=.000, h^{2}=.10$. Las comparaciones múltiples indican que existen diferencias estadísticamente significativas, en todos los factores, entre el poco dominio y un gran dominio, que para 535 gl: PE: $\mathrm{t}=-4.812(p=.000)$; FUP: $\mathrm{t}=-4.565(p=.000) ; \mathrm{y}$ AO: $\mathrm{t}=-4.143(p=.000)$.

Valores que permiten rechazar todas las hipótesis nulas formuladas con un riesgo alfa de equivocarnos igual o inferior al .05; en consecuencia podemos decir que el dominio técnico que el alumnado indica tener de los ordenadores influye en la percepción de éxito, la percepción de facilidad de uso de Moodle y en la autoeficacia respecto al ordenador.

En relación, al dominio técnico que el alumno indica tener de los medios audiovisuales, la hipótesis nula (H0) formulada se refiere a si éste no tiene una influencia significativa, con un riesgo alfa de equivocarnos del .05 y aplicando el estadístico de ANOVA, en la percepción de éxito $\mathrm{F}(10.535)=3.477$ y $p=.000, \mathrm{~h}^{2}=.06$; la percepción de facilidad de uso $\mathrm{F}(10.535)=6.119$ y $p=.000, \mathrm{~h}^{2}=.11$; $\mathrm{y}$ en la autoeficacia respecto al ordenador $\mathrm{F}(10.535)=4.644$ y $p=.000, \mathrm{~h}^{2}=.08$. Las comparaciones múltiples indican que existen diferencias estadísticamente significativas, en todos los factores, entre el poco dominio y bastante dominio, que para 535 gl: PE: $\mathbf{t}=-3.407$ ( $p=.038)$; FUP: $\mathrm{t}=-3.350(p=.046) ;$ y AO: $\mathbf{t}=-4.254(p=.001)$.

Estos resultados permiten rechazar todas las hipótesis nulas formuladas con un riesgo alfa de equivocarnos igual o inferior al .05; en consecuencia, podemos decir que el dominio técnico que el alumnado indica tener de los medios audiovisuales influye en la percepción de éxito, la percepción de facilidad de uso de Moodle y en la autoeficacia respecto al ordenador.

Finalmente, el estudio de las hipótesis nulas que se desprenden de las dimensiones de los factores percepción de éxito, percepción de facilidad de uso de Moodle y autoeficacia respecto al ordenador en relación al soporte técnico, que hacían referencia a la no existencia de influencias, aplicado el coeficiente de correlación de Pearson, con un riesgo alfa de equivocarnos inferior al .01, desprende que el soporte técnico, afecta positiva y significativamente sobre la percepción de éxito ( $R=.348$ y p=.000), la autoeficacia con el ordenador $(\mathrm{R}=.374$ y $p=.000)$, y la percepción de facilidad de uso de Moodle $(\mathrm{R}=.224$ y $p=.000)$. 
El análisis de las hipótesis nulas (H0) que se desprenden de las dimensiones del modelo TAM formulado (figura 2), que hacían referencia a la no existencia de influencias con un riesgo alfa de equivocarnos del .05, aplicamos el coeficiente de correlación de Pearson (tabla 4).

Tabla 4

Coeficiente de correlación de Pearson

\begin{tabular}{lccccc}
\hline & PE & AO & FUP & A & US \\
\hline PE & 1 & $.488^{* *}$ & $.444^{* *}$ & $.622^{* *}$ & $.339^{* *}$ \\
AO & 1 & $.591^{* *}$ & $.480^{* *}$ & $.275^{* *}$ \\
FUP & & 1 & $.456^{* *}$ & $.275^{* *}$ \\
A & & & & 1 & $.393^{* *}$ \\
US & & & & & 1 \\
\hline
\end{tabular}

Nota ${ }^{* *}$ significativo a alfa igual a .01 .

Los coeficientes de correlación de Pearson obtenidos, permiten obtener dos grandes conclusiones: rechazar todas las $\mathrm{H} 0$ formuladas, con un riesgo alfa de equivocarnos inferior al .01. En consecuencia, podemos señalar que:

- La percepción de éxito, afecta positiva y significativamente sobre la autoeficacia con el ordenador, la percepción de facilidad de uso de Moodle, con la actitud y con el uso del sistema.

- La autoeficacia con el ordenador, afecta positiva y significativamente la percepción de facilidad de uso de Moodle, con la actitud y con el uso del sistema.

- La percepción de la facilidad de uso de Moodle, afecta positiva y significativamente con la actitud y con el uso del sistema.

- Y que la actitud hacia el uso de Moodle afecta positiva y significativamente en el uso que se haga de la misma.

Y en segundo lugar, que el signo positivo obtenido en todas las correlaciones indican que las variables contrastadas aumentan cuando una de ella lo hace.

El resultado del modelo estructural lo encontramos en la tabla 5 y en la figura 3. La tabla 5 nos permite conocer los valores de coeficiente de determinación $\mathrm{R}$ para cada una de las variables endógenas del sistema TAM. Tabla y figura que nos indican la alta importancia estructural entre las variables del estudio, relaciones que en todos los casos se establecen de manera de significativa; por otra parte el valor $\mathrm{R}$ nos indica que tanto la "percepción de facilidad de uso", como la "percepción de éxito" y la "autoeficacia respecto al ordenador", están bien explicadas en un $66 \%$, $41 \%$ y $58 \%$ respectivamente.

Los valores al mismo tiempo ratifican la significación de la hipótesis H10, H11 y H12 formuladas respecto al soporte técnico, las H18, H20 y H23 referidas a la percepción de 
facilidad de uso; las H22 y H24 respecto a la significación de la percepción de éxito en las actitudes e intención de uso, y la H21 respecto a la actitud por el uso y la intención de uso.

Tabla 5

Efectos de las variables sobre la aceptación de Moodle

\begin{tabular}{lcccccccc}
\hline & $\begin{array}{c}\text { Percepción } \\
\text { facilidad de } \\
\text { uso }\end{array}$ & \multicolumn{2}{c}{$\begin{array}{c}\text { Percepción de } \\
\text { exito }\end{array}$} & \multicolumn{2}{c}{$\begin{array}{c}\text { Autoeficacia } \\
\text { respecto al } \\
\text { ordenador }\end{array}$} & \multicolumn{2}{c}{$\begin{array}{c}\text { Uso del } \\
\text { sistema }\end{array}$} \\
\hline & ED. & EI. & ED. & EI. & ED. & EI. & ED. & EI. \\
\hline $\begin{array}{l}\text { Soporte Técnico } \\
\text { Percepción facilidad }\end{array}$ & $4.17^{* *}$ & & $2.18^{* *}$ & & $.61^{* *}$ & & & $.49^{*}$ \\
$\begin{array}{l}\text { de uso } \\
\text { Percepción de éxito }\end{array}$ & & $1.39^{* *}$ & $.78^{* *}$ & $1.80^{* *}$ & $.55^{*}$ & $2.44^{* *}$ & $.41^{*}$ \\
$\begin{array}{l}\text { Autoeficacia respecto } \\
\text { al ordenador }\end{array}$ & & & & $1.49^{* * *}$ & $.672^{* *}$ & $1.66^{* *}$ & $.60^{*}$ \\
$\mathrm{R}^{2}$ & & & & & & & $1.67^{* *}$ & $.59^{*}$ \\
\hline
\end{tabular}

$\mathrm{p}<.05, "$ " $\mathrm{p}<.01$

$\mathrm{ED}=$ Efecto directo, $\mathrm{EI}=$ Efecto indirecto

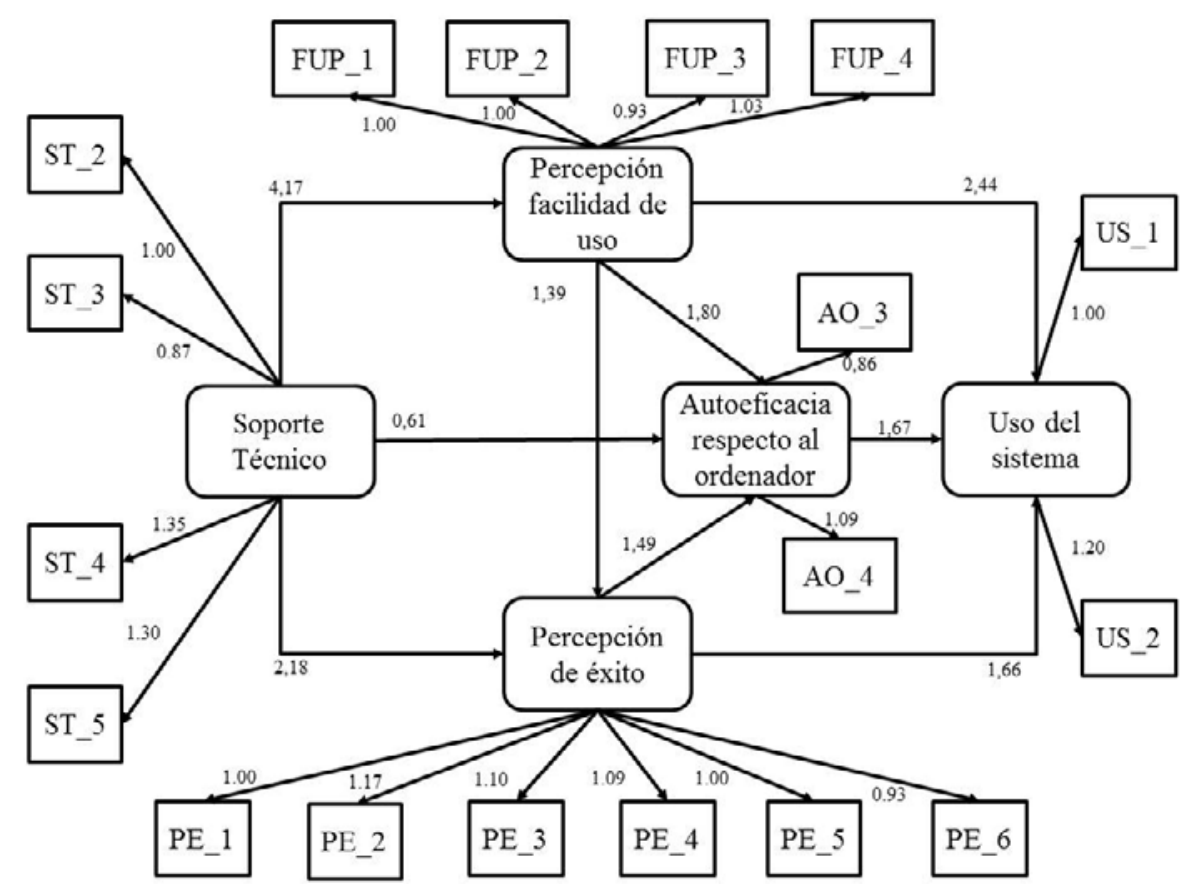

Figura 3. Modelo Estructural 


\section{Discusión y conclusiones}

Nuestro estudio permite obtener diferentes conclusiones:

a) En índice de fiabilidad del instrumento, tanto de forma general como en las diferentes dimensiones que lo conformaban nos permite señalar que se cuenta con un instrumento fiable para el análisis del modelo TAM aplicado al uso del Moodle. Por otra parte, nuestros valores fueron muy superiores a los alcanzados por Arteaga y Duarte (2010) con alumnos de la Universidad de Huelva.

b) El modelo TAM elaborado se presenta como un buen predictor para explicar la actitud hacia la Moodle y el uso de esta plataforma para ser utilizada en la educación, lo que pone de manifiesto la significación del modelo inicialmente formulado por Davis (1989). Resultados que se encuentran en la misma dirección de los alcanzados por Arteaga y Duarte (2010).

c) Los hallazgos de nuestro trabajo coinciden con los obtenidos por PadillaMeléndez et al. (2015), que llegan a señalar que el entrenamiento, formación, de los estudiantes en la plataforma no tenía un impacto directo en el uso de la plataforma Moodle.

d) El soporte técnico de la plataforma Moodle posee relación positiva con la percepción de éxito, la percepción en la facilidad de uso de Moodle y, la autoeficacia respecto al ordenador. Lo que confirma las aportaciones de diferentes autores al señalar que la incorporación de cualquier tecnología, debe estar acompañada por medidas organizativas que faciliten su utilización tanto por docentes como por discentes, como pone claramente de manifiesto el meta-análisis de investigaciones sobre el uso de las TIC por los docentes recientemente realizado por APS Group Scotland (2015).

e) El haber realizado acciones formativas para el aprendizaje de alguna tecnología de la información y comunicación no determina la percepción de éxito, la percepción en la facilidad de uso de Moodle, ni la autoeficacia respecto al ordenador. Hecho que pudiera parecer contradictorio con lo aportado en diferente literatura científica (Roblizo \& Cózar, 2015), en nuestro caso pensamos que ello era debido al bajo porcentaje de estudiantes que indicaron que sí la habían recibido. Ya que si nos hemos encontrado que, tanto el dominio técnico que el alumnado indica tener de los ordenadores, como de los medios audiovisuales, si tenían un efecto positivo sobre percepción de éxito, la percepción en la facilidad de uso de Moodle y, la autoeficacia respecto al ordenador. Resultados que coinciden con los hallazgos de Tarhini et al. (2014), donde la experiencia modela la facilidad de uso y la utilidad percibida hacia la tecnología, y Buchanan, Joban y Porter (2014) sobre la significación de la autoeficacia percibida por parte del estudiante en la interacción que realiza en Internet.

f) El género del estudiante no determina la percepción de éxito, la percepción en la facilidad de uso de Moodle, ni la autoeficacia respecto al ordenador, lo que coincide con los hallazgos de no relación entre género y tecnologías de otros autores (Bullón, Cabero, Llorente, Machuca, Machuca \& Marín., 2009; Cabero et 
al., 2009; Hohlfeld et al., 2013). Y posiblemente nos sugieran que las diferencias no se encuentren tanto en la competencia para el uso, sino más en los diferentes tipos de usos que hacen, como han encontrado Lim y Meier (2011).

Para finalizar señalar que futuras investigaciones pueden trabajar con otras variables predictoras, que no han sido contempladas en el presente trabajo, como son: la diversidad de usos de las TIC que realizan los sujetos, la calidad y tipología del soporte ofrecido por los docentes y los pares de los estudiantes para la comprensión del sistema Moodle, o la accesibilidad y estética del sistema

\section{Referencias}

Aguilar-Luzón, M.C., Berrios-Martos, M.P., \& López-Zafra, E. (2012). El uso de sistemas informáticos en el estudio de las materias universitarias: aplicación de la teoría de la conducta planificada y de la teoría de la aceptación de las tecnologías. Estudios de Psicología, 33(2), 179-190.

Ajzen, I., \& Fishbein, M (1980). Understanding attitudes and predicting social behavior. Englewood Cliffs, NJ: Prentice-Hall.

Alharbi, S., \& Drew, S. (2014). Using the Technology Acceptance Model in Understanding Academics' Behavioural Intention to Use Learning Management Systems. International Journal of Advanced Computer Science and Applications, 5(1), 143-155.

APS Group Scotland (2015). Literature Review on the Impact of Digital Technology on Learning and Teaching. Social Research, 2, Recuperado de http://www.gov.scot/ Resource/0048/00489303.pdf

Arenas, J., Peral-Peral, B., \& Ramón-Jerónimo, M. (2014). Gender in the Elderly Internet Users. En I. Gil Pechuán, D. Palacios-Marqués, M. Peris-Ortiz, E. Vendrell, C. FerriRamírez (Eds.), Strategies in E-Business (pp. 67-76). doi: 10.1007/978-1-4614-8184-3.

Arteaga, R., \& Duarte, A. (2010). Motivational factors that influence the acceptance of Moodle using TAM. Computers in Human Behavior, 26(6), 1632-1640. doi: http:// dx.doi.org/10.1016/j.chb.2010.06.011.

Bandura, A. (1990). Perceived self-efficacy in the exercise of personal agency. Revista Española de Pedagogía, 187, 397-427.

Bao, Y., Xong, T., Hu, Z., \& Kibelloh, M. (2013). Exploring gender differences on general and specific computer self-efficacy in mobile learning adoption. Journal Educational Computing Research, 49(1) 111-132. doi: http://dx.doi.org/10.2190/EC.49.1

Buchanan, T., Joban, S., \& Porter, A. (2014). Internet self-efficacy does not predict student use of Internet-mediated educational technology. Research in Learning Technology, 22, 1-14. doi: http://dx.doi.org/10.3402/rlt.v22.19585.

Bullón, P., Cabero, J., Llorente, Maㅡ C., Machuca, Maㅡ C., Machuca, G., \& Marín, V. (2009), Competencias tecnológicas del profesorado de la Facultad de Odontología de la Universidad de Sevilla. Sevilla: GID

Cabero, J., \& Barroso, J. (2016). The educational possibilities of Augmented Reality. NAER, 5(1). 44-50. doi: http://dx.doi.org/10.7821/naer.2016.1.140. 
Cabero, J., Leal, F., Andrés, F., \& Llorente, M.C. (2009). La alfabetización digital de los alumnos universitarios mexicanos. Enseñanza E Teaching, 27(1), 41-59.

Colorado, A., Marín, V., \& Zavala, Z. (2016). Impacto del grado de apropiación tecnológica en los estudiantes de la Universidad Veracruzana. IJERI, Revista internacional de Investigación e Innovación Educativa, 5, 124-137.

Chen, B., Sivo, S., Seilhamer, R., Sugar, A., y Mao, J. (2013). User acceptance of mobile technology: a campus-wide implementation of blackboard's mobile ${ }^{\mathrm{TM}}$ learn application. Journal Educational Computing Research, 49(3) 327-343. doi: http:// dx.doi.org/10.2190/EC.49.3.c.

Chen, Y-H., \& Chengalur-Smith, I. (2015). Factors influencing students' use of a library Web portal: Applying course-integrated information literacy instruction as an intervention. Internet and Higher Education, 26, 42-55. doi: http://dx.doi. org/10.1016/j.iheduc.2015.04.005.

Cheng, Y., Lou, S., Kuo, S., \& Shih, R. (2013). Investigating elementary school students' technology acceptance by applying digital game-based learning to environmental education. Australasian Journal of Educational Technology, 29(1), 96-110. doi: http:// dx.doi.org/10.14742/ajet.65.

Davis, F. D. (1989), Perceived usefulness, perceived ease of use, and user acceptance of information technology. MIS Quarterly, 13(3), 319-340. doi:10.2307/249008.

Escobar, T., Carvajal, E., \& Monge, P. (2014). Factors that influence the perceived advantages and relevance. Australasian Journal of Educational Technology, 30(2), 136-155. doi: http://dx.doi.org/10.14742/ajet.585.

Gámiz, V., \& Gallego, M.J. (2016), Modelo de análisis de metodologías didácticas semipresenciales en Educación Superior. Educación XX1, 19(1), 39-61. doi: http:// dx.doi.org/10.5944/educxx1.15577

García-Lirios. C. (2012). Estructura híbrida de los determinantes sociodemográficos del consume electrónico. Revista de Psicología GEPU, 3(2), 43-53.

George, D., \& Mallery, P. (2009). SPSS for Windows step by step: A simple guide and reference.17.0 Update. Boston: Allyn \& Bacon.

González-Bravo, L., \& Valdivia-Peralta, M. (2015). Posibilidades para el uso del modelo de aceptación de la tecnología (TAM) y de la teoría de los marcos tecnológicos para evaluar. Revista Electrónica Educare, 19(2), 181-196. doi: http://dx.doi.org/10.15359/ ree.19-2.11.

Hernández, R., Fernández, C., \& Baptista, P. (2014). Metodología de la investigación. (6ª . ed.). México: McGraw Hill Interamericana.

Herrero, R., González, I., \& Marín, V. (2015). Formación centrada en adquisición de competencias. El caso del grado de educación primaria. Revista de Ciencias Sociales, XXI (4), 461-478.

Ho, L-H., Hung, Ch-L., \& Chen, H-Ch. (2013). Using theoretical models to examine the acceptance behavior of mobile phone messaging to enhance parent-teacher interaction. Computers $\mathcal{E}$ Education, 61, 105-114. doi:10.1016/j.compedu.2012.09.009.

Hohlfeld, T., Ritzhaupt, A., \& Barron, A. (2013). Are gender differences in perceived and demonstrated technology literacy significant? It depends of the model. Education Technology Research and Development, 61(4), 639-663. doi: http://dx.doi. org/10.1007/s11423-013-9304-7. 
Hsiao, C.H., \& Yang, C. (2011). The intellectual development of the technology acceptance model: A co-citation analysis. International Journal of Information Management, 31(2), 128-136. doi: http://dx.doi.org/10.1016/j.ijinfomgt.2010.07.003.

Jin, C.H. (2014). Adoption of e-book among college students: The perspective of an integrated TAM. Computers in Human Behavior, 41, 471-477. doi: http://dx.doi. org/10.1016/j.chb.2014.09.056.

Jou, M., \& Wang, J. (2013). Observations of achievement and motivation in using cloud computing driven. Computers in Human Behavior, 29(2), 364-369. doi: http:// dx.doi.org/10.1016/j.chb.2012.08.001.

Kang, M., \& Shin, W. (2015). An Empirical Investigation of Student Acceptance of Synchronous E-Learning in an Online University. Journal of Educational Computing Research, 52(4), 475-495. doi:10.1177/0735633115571921.

Kumar, S., \& Kumar, J. (2013). Technology acceptance model for the use learning through websites among students in Oman. International Arab Journal of e-Technology, $3(1), 44-49$.

Lee, D., \& Lehto, M. (2013). User acceptance of YouTube for procedural learning: An extension of the Technology Acceptance Model. Computers E Education, 61, 193208. doi: http://dx.doi.org/10.1016/j.compedu.2012.10.001

Lim, K., \& Meier, E. (2011). Different but similar: computer use patterns between young Korean males and females. Education Technology Research Development, 59(4), 575-592.

Liu, I-F, Chang, M., Sun, Y., Wible, D., \& Kuo, Ch-H. (2010). Extending the TAM model to explore the factors that affect Intention to Use an Online Learning Community. Computers E Education, 54, 600-610. doi: http://dx.doi.org/10.1016/j. compedu.2009.09.009.

López-Bonilla, L.M., \& López-Bonilla, J.M. (2011). Los modelos de adopción de tecnologías de la información desde el paradigma actitudinal. Cadernos EBAPE. BR, 9(1), 177-197.

Marín, V., Sampedro, B.E., \& Muñoz, J.M. (2015). ¿Son adictos a las redes sociales los estudiantes universitarios? Revista Complutense de Educación, 26, 233-251. doi:10.5209/rev_RCED.2015.v26.46659.

Marín, V., Ramírez, M., \& Maldonado, G.A. (2016). Valoraciones del profesorado universitario sobre la integración de las TIC en el aula. EDMETIC, Revista de Educación Mediática y TIC, 5(1), 177-200. doi: https://doi.org/10.21071/edmetic.v5i1.4022

doi: http://dx.doi.org/10.6018/rie.36.2.292951

Mateo, J. (2004). La investigación ex post-facto. En R. Bisquerra (coord.), Metodología de la investigación educativa (pp.195-230). Madrid: La Muralla.

Mohammadi, H. (2015). Investigating users' perspectives on e-learning: an integration of TAM and IS success model. Computers in Human Behavior, 45, 359-374. doi: http:// dx.doi.org/10.1016/j.chb.2014.07.044.

Ngai, E. Poon, J., \& Chan, Y. (2007). Empirical examination of the adoption of WebCT using TAM. Computers \& Education, 48(2), 250-267. doi:10.1016/j. compedu.2004.11.007.

O’Dwyer, L., \& Bernauer, J. (2014). Quantitative research for the qualitative researcher. California: Sage. 
Olaoluwakotansibe, A. (2013). Interactive digital technologies' use in Southwest Nigerian universities. Education Technology Research Development, 61(2), 333-357.

Padilla-Meléndez, A., Del Águila-Obra, A. R., \& Garrido-Moreno, A. (2015). Empleo de Moodle en los procesos de enseñanza-aprendizaje de Dirección de Empresas: nuevo perfil del estudiante en el EEES. Educación XX1, 18(1), 125-146. doi: http:// dx.doi.org/10.5944/educXX1.18.1.12314.

Park, S., Nam, M-W., \& Cha, S. B. (2012). University students' behavioral intention to use mobile learning: Evaluating the technology acceptance model. British Journal of Educational Technology, 43(4), 592-605. doi:10.1111/j.1467-8535.2011.01229.x.

Park, T., Saplan, V., \& Jaegal, D. (2012). Predicting knowledge sharing intentions in the public sector: comparing TAM with TPB. International Review of Public Administration, 17(2), 93-120.

Peral-Peral, B., Arenas, J., \& Ramón-Jerónimo, M.A. (2014). Technology Acceptance Model y mayores: ¿la educación y la actividad laboral desarrollada son variables moderadoras? Revista Española de Investigación en Marketing ESIC, 18(1), 43-56. doi: http://dx.doi.org/10.1016/S1138-1442(14)60005-X.

Persico, D., Manca, S., \& Pozzi, F (2014). Adapting the Technology Acceptance Model to evaluate the innovative potential of e-learning systems. Computers in Human Behavior, 30, 614-622. doi: http://dx.doi.org/10.1016/j.chb.2013.07.045.

Roblizo, M., \& Cózar, R. (2015). Usos y competencias en TIC en los futuros maestros de educación infantil y primaria: hacia una alfabetización tecnológica real para docentes. Pixel-Bit. Revista de Medios y Educación, 47, 23-39. doi: http://dx.doi. org/10.12795/pixelbit.2015.i47.02.

Sánchez-Franco, M. (2010). WebCT-The quasimoderating effect of perceived affective quality on an extending Technology Acceptance Model. Computers E Education, 54, 37-46. doi: http://dx.doi.org/10.1016/j.compedu.2009.07.005.

Schoonenboom, J. (2014). Using an adapted, task-level technology acceptance model to explain why instructors in higher education intend to use some learning management system tools more than others. Computers $\mathcal{E}$ Education, 71, 247-256. doi: http://dx.doi.org/10.1016/j.compedu.2013.09.016.

Silva, P., Pimentel, V., \& Soares, J. (2012). A utilização do computador na educação: aplicando o Technology Acceptance Model (TAM). Biblionline, João Pessoa, 8, 263-272.

Tarhini, A., Hone, K. \& Liu, X. (2014). A cross-cultural examination on of the impact of social, organizational and individual factors on educational technology acceptance between British and Lebanese university students. British Journal of Educational Technology, 46(4), 739-755. doi: http://dx.doi.org/10.1111/bjet.12169.

Teo, T., Fan, X., \& Du, J. (2015). Technology acceptance among pre-service teachers: Does gender matter? Australasian Journal of Educational Technology, 31(3), 235-251.

Terzis, V., \& Economides, A. A. (2011). The acceptance and use of computer based assessment. Computers $\mathcal{E}$ Education, 56, 1032-1044. doi: http://dx.doi.org/10.1016/j. compedu.2010.11.017.

Valencia, A., Benjumea, M., \& Rodríguez, V. (2014). Intención de uso del e-learning en el programa de Administración Tecnológica desde la perspectiva del modelo de aceptación tecnológica. Revista Electrónica Educare, 18(2), 247-264. doi: http:// dx.doi.org/10.15359/ree.18-2.13. 
Wai-tsz, R., Chi-kin, J., Chang, Ch., Zhang, Z., \& Chiu, A. (2014). Digital teaching portfolio in higher education: Examining colleagues' perceptions to inform implementation strategies. Internet and Higher Education, 20, 60-68. doi: http:// dx.doi.org/10.1016/j.iheduc.2013.06.003.

Yong, L.A., Rivas, L.A., \& Chaparro, J. (2010). Modelo de aceptación tecnológica (TAM): un estudio de la influencia de la cultura nacional y del perfil del usuario en el uso de las TIC. Innovar, 20(36), 187-204.

Fecha de recepción: 2 de mayo de 2017

Fecha de revisión: 15 de mayo de 2017

Fecha de aceptación: 22 de marzo de 2018 
